\title{
Intracranial Leptomeningeal Lesion
}

National Cancer Institute

\section{Source}

National Cancer Institute. Intracranial Leptomeningeal Lesion. NCI Thesaurus. Code C84358.

A non-neoplastic or neoplastic pathologic process affecting the leptomeninges of the brain. 